\title{
Inflammation: The Good or the Bad Guy?
}

\section{Maria Benito}

IPN Communications, Dublin, Ireland

*Corresponding Author: Maria Benito, Clinical Medicine, IPN Communications, Dublin, Ireland.

Received: July 01, 2019; Published: July 11, 2019

DOI: 10.31080/ASMS.2019.03.0351

\begin{abstract}
Acute inflammation is a self-regulated mechanism that protect against insults or tissue damage. Dysregulation of this process can make the inflammation chronic and further damage normal tissue function.
\end{abstract}

Keywords: Inflammation; Resolution; Inflammatory Process; Dysregulation; Neutrophils; Macrophages

\section{Abbreviations}

LTB4: Leukotriene B4); LXA4: Lipoxin A4; PGD2: Prostaglandin D2; PGE2: Prostaglandin E2; PGJ2: Prostaglandin J2; RvE1: Resolvin E1; TGF $\beta$ : Transforming Growth Factor Beta; TNF-1?: Tumour Necrosis Factor-Alpha.

Benefit that the body receives by inflammation

Inflammation is a process that protects tissues from many types of insults, from foreign pathogens attacks to autoimmune assault. To confront these harms, the body responds with acute inflammation that aims to get rid of the invader through the immune system (lymphocytes -T cells, B cells, and natural killer cells-, macrophages, dendritic cells and neutrophils).

Upon injury, neutrophils infiltrate the tissue and start secreting cytokines and mediators to recruit other immune cells that also secrete more inflammatory mediator perpetuating the inflammation. Prostaglandin E2 $\left(\mathrm{PGE}_{2}\right)$ and tumor necrosis factor $\alpha(\mathrm{TNF} \alpha)$ are some of the mediators involved in the initiation process $[1,2]$.

Inflammation follows a sequence of induction, a peak of inflammation, and finally a phase of resolution to eliminate the injury. The inflammatory process has an internal cost in homeostasis and alteration of the normal tissue function.

Once the damage has been overcome, the resolution process starts with the cease of neutrophils recruitment to the inflamed tissue, switching the production from pro-inflammatory agents such as $\mathrm{PGE}_{2}$ and Leukotriene B4 (LTB4) to pro-resolving lipid mediators such as $\mathrm{PGD}_{2}$, PGJ2, lipoxin A4 (LXA4), resolvin E1 (RvE1), protectin D1, and maresin-1 [3]. Then, it is necessary the removal of neutrophils through apoptosis induced by death ligands produced by macrophages $[4,5]$ or transforming growth factor beta (TGF $\beta$ ) produced by regulatory $\mathrm{T}$ cells [6]. Macrophages need to change their role -from M1 to M2- acquiring anti-inflammatory and proresolving functions [7], and clear the apoptotic neutrophils through efferocytosis.

\section{Damage caused by chronic inflammation}

However, sometimes this process of inflammation is dysregulated and instead of resolving becomes chronic leading to other pathologies. Thus, although inflammation is a beneficial process designed to eradicate threats to the organism, a dysregulation of inflammation -either in magnitude or duration- contributes to multiple pathologies [8] such as rheumatoid arthritis, Crohn's disease, cancer, vascular diseases and asthma [9-12]. In extreme cases, the dysregulated inflammatory reaction can cause inflammatory tissue damage and sepsis $[13,14]$

Recently, mechanisms tissue and disease-specific that funnel the resolution of inflammation in arthritis, colitis, and asthma have been identified [12]. These pathways -as well as the discovery of new players that may play a role in inflammation- can be a potential target to reverse chronic inflammation in inflammatory diseases. 


\section{Conclusion}

Despite the benefit of acute inflammation to defend against pathogens or insults, chronic inflammation can cause more damage and contribute to the development of additional pathologies.

\section{Bibliography}

1. Kawahara K., et al. "Prostaglandin E2-induced inflammation: Relevance of prostaglandin E receptors”. Biochimica et Biophysica Acta 1851.4 (2015): 414-421.

2. Bradley JR. "TNF-mediated inflammatory disease". The Journal of Pathology 214.2 (2008): 149-160.

3. McGrath E., et al. "TNF-related apoptosis-inducing ligand (TRAIL) regulates inflammatory neutrophil apoptosis and enhances resolution of inflammation". Journal of Leukocyte Biology 90.5 (2011): 855-865.

4. Brown SB and Savill J. "Phagocytosis triggers macrophage release of Fas ligand and induces apoptosis of bystander leukocytes". Journal of Immunology 162.1 (1999): 480-485.

5. Gagliani N., et al. "TH17 cells transdifferentiate into regulatory T cells during resolution of inflammation". Nature 523.7559 (2015): 221-225.

6. Levy BD., et al. "Lipid mediator class switching during acute inflammation: signals in resolution". Nature Immunology 2.7 (2001): 612-619.

7. Hannemann N., et al. "The AP-1 transcription factor c-Jun promotes arthritis by regulating cyclooxygenase- 2 and arginase-1 expression in macrophages". Journal of Immunology 198.9 (2017): 3605-3614.

8. Fullerton JN and Gilroy DW. "Resolution of inflammation: a new therapeutic frontier". Nature Reviews Drug Discovery 15.8 (2016): 551-567.

9. Schett G., et al. "How cytokine networks fuel inflammation: toward a cytokine-based disease taxonomy". Nature Medi- cine 19.7 (2013): 822-824.

10. McInnes IB and Schett G. "Pathogenetic insights from the treatment of rheumatoid arthritis". Lancet 389.10086 (2017): 2328-2337.

11. Nielsen $\mathrm{OH}$ and Ainsworth MA. "Tumor necrosis factor inhibitors for inflammatory bowel disease". The New England Journal of Medicine 369 (2013): 754-762.

12. Schett G and Neurath MF. "Resolution of chronic inflammatory disease: universal and tissue-specific concepts". Nature Communications 9 (2018): 3261.

13. Nathan C. "Points of control in inflammation". Nature 420.6917 (2002): 846-852.

14. Nathan C and Ding A. "Nonresolving inflammation". Cell 140.6 (2010): 871-882.

\section{Volume 3 Issue 8 August 2019} (C) All rights are reserved by Maria Benito. 